\title{
let-7d-3p is associated with apoptosis and response to neoadjuvant chemotherapy in ovarian cancer
}

\author{
RAÚL GARCÍA-VÁZQUEZ ${ }^{1}$, DOLORES GALLARDO RINCÓN², ERIKA RUIZ-GARCÍA ${ }^{2}$, \\ ABELARDO MENESES GARCÍA ${ }^{2}$, OLGA N. HERNÁNDEZ DE LA CRUZ ${ }^{3}$, HORACIO ASTUDILLO-DE LA VEGA ${ }^{4}$, \\ DAVID ISLA-ORTIZ ${ }^{5}$, LAURENCE A. MARCHAT ${ }^{1}$, YARELY M. SALINAS-VERA ${ }^{3}$, ÁNGELES CARLOS-REYES ${ }^{6}$, \\ SULLIVAN LÓPEZ-GONZÁLEZ ${ }^{6}$, ROSALIO RAMOS-PAYAN ${ }^{7}$ and CÉSAR LÓPEZ-CAMARILLO ${ }^{3}$
}

\footnotetext{
${ }^{1}$ Molecular Biomedicine Program and Biotechnology Network, Instituto Politecnico Nacional, México City 07320;

${ }^{2}$ Translational Medicine Laboratory, National Institute of Cancerology, México City 14080;

${ }^{3}$ Genomics Sciences Program, Autonomous University of Mexico City, México City 03100;

${ }^{4}$ Laboratory of Translational Cancer Research and Cellular Therapy, National Medical Center 'Century XXI', México City 06720; ${ }^{5}$ Department of Oncologic Surgery, National Institute of Cancerology, México City 14080;

${ }^{6}$ Laboratory of Lung Cancer, National Institute of Respiratory Diseases 'Ismael Cosio Villegas', Mexico City 14080;

${ }^{7}$ Sciences Faculty, Autonomous University of Culiacan, Culiacan Sinaloa 80040, Mexico
}

Received September 1, 2017; Accepted March 30, 2018

DOI: $10.3892 / o r .2018 .6366$

\begin{abstract}
Altered expression of microRNAs contributes to the heterogeneous biological behavior of human malignancies and it may correlate with the clinical pathological features of patients. The let-7 microRNA family is frequently downregulated in human cancers and its aberrant expression may be a useful marker for prediction of the clinical response to therapy in patients. In the present study, we analyzed the expression of three members of the let-7 family (let-7a-3p, let-7d-3p and let-7f), which remains largely uncharacterized in ovarian cancer tissues. We also investigated the function of let-7d-3p in the apoptosis and sensitization to chemotherapy in ovarian cancer cells. Our data from stem-loop quantitative RT-PCR showed that expression of let-7a-3p and let-7d-3p, but not let-7f, was significantly $(\mathrm{P}<0.04)$ upregulated in ovarian tumors relative to that noted in normal ovarian tissues. Markedly, an increased expression of let-7d-3p (also known as let-7d-3*) was associated with positive response to carboplatin/paclitaxel treatment in ovarian cancer patients. To investigate the biological relevance of let-7d-3p, we knocked down its expression in SKOV-3 ovarian cancer cell line using antagomiRs. Loss of function analysis showed that inhibition of let-7d-3p significantly $(\mathrm{P}<0.05)$ impaired cell proliferation and activated apoptosis. In contrast, scratch/wound healing and
\end{abstract}

Correspondence to: Dr César López-Camarillo, Genomics Sciences Program, Autonomous University of Mexico City, San Lorenzo 290, Col del Valle Sur, Mexico City 03100, Mexico

E-mail: genomicas@yahoo.com.mx

Key words: ovarian cancer, let-7, apoptosis, chemotherapy response, neoadjuvant therapy
Transwell chamber assays showed that migration and invasion abilities were not affected in the let-7d-3p-deficient SKOV-3 cancer cells. Notably, Annexin V assays showed a significant $(\mathrm{P}<0.05)$ increase in cell death of cancer cells treated with the let-7d-3p inhibitor plus carboplatin indicating a synergistic effect of the drug with antagomiR therapy. Gene ontology classification of predicted targets of let-7d-3p identified a number of genes involved in cellular pathways associated with therapy resistance such as ABC transporters, HIF-1, RAS and ErbB signaling. In summary, our findings showed that inhibition of let-7d-3 activates apoptosis and that its upregulation is associated with a positive response of ovarian cancer patients to carboplatin/paclitaxel chemotherapy.

\section{Introduction}

MicroRNAs (miRNAs) are evolutionarily conserved small non-coding RNAs of 25 nucleotides in length that function as negative regulators of gene expression by either inhibiting translation or inducing degradation of specific messenger RNA targets (1). miRNA expression could be disturbed by carcinogenic agents, chemotherapy and diverse external stimuli, which could impact genetic and epigenetic programs contributing to the heterogeneous biological behavior of tumors. For instance, long-term arsenic exposure of keratinocytes resulted in the upregulation of miR-21, miR-200a and miR-141, which were found to be involved in the development of melanoma, thus they could be promising early biomarkers of skin cancer (2). Importantly, changes in the abundance of miRNAs in tumors may correlates with clinical and pathological features of patients. For example, the downregulation of tumor-suppressor miR-198 and upregulation of MSLN, OCT-2, PBX-1 and VCP in pancreatic tumors were associated with the poor survival of patients (3). Consequently, miRNAs represent novel prognostic biomarkers and promising translational targets in 
cancer therapy. Particularly, the let-7 family of miRNAs are frequently downregulated in diverse types of cancers. let-7 is a major regulator of differentiation, pluripotency and apoptosis in eukaryotic cells (4-6). In cancer cells, let-7 targets multiple oncogenes involved in the deregulation of the cell cycle, cell division, proliferation, angiogenesis and apoptosis (7). Markedly, experimental restoration of normal expression levels of let-7 in cancer cells prevents tumorigenesis indicating that it acts as a bona fide tumor suppressor. These findings suggest that let-7 members can be used as molecular tools and markers in cancer therapeutics.

Epithelial ovarian cancer (EOC) is a highly metastatic disease with the highest mortality rate of all gynecologic cancers (8). More than $90 \%$ ovarian cancers are classified as epithelial whereas the remaining most frequent histotypes are serous, endometrioid, clear-cell and mucinous. Until recently these malignancies were considered as derived from ovarian surface epithelium. The different ovarian cancer histotypes are characterized by altered genomic and epigenetic patterns, which greatly impact oncogenic signaling pathways, biological behavior and clinical outcome (9). Conventional treatment of ovarian cancer is based on surgery and chemotherapy. Platinum-based agents including cisplatin and carboplatin represent the first-line agents for patients with advanced ovarian cancer (10). Randomized controlled clinical trials established that this therapeutic regimen yields 5-year survival rate from 30 to $92 \%$; and 40 to $60 \%$ complete responses depending on the spread of disease at time of diagnosis (11). Although most patients with ovarian cancer exhibit response to combination chemotherapy of platinum salts, many patients develop resistance and relapse with a median progression-free survival of only 18 months (12). However, although cisplatin resistance mechanisms have been studied for decades, the genes and factors involved in this adverse cellular event have not been fully identified (13). The scenario is worse as no molecular predictors of clinical response to therapy are currently in use, although several cellular factors are becoming increasingly studied (14).

Neoadjuvant chemotherapy has been recognized as a reliable therapeutic strategy in patients with unresectable EOC. Some advances in the study of cellular events leading to proper response to neoadjuvant chemotherapy have been reported (15). However, the potential role of miRNAs in neoadjuvant chemotherapy has not been fully explored in ovarian cancer. In the present study, we investigated the changes in expression of let-7 family members in order to evaluate whether they have a prognostic role in EOC patients who received neoadjuvant chemotherapy. In addition, we provide experimental evidence concerning the role of let-7d-3p in apoptosis and sensitization of ovarian cancer cells to chemotherapy.

\section{Materials and methods}

Cell lines. Human ovarian cancer cell line SKOV-3 was obtained from the American Type Culture Collection (ATCC; Manassas, VA, USA) and grown in Dulbecco's modified Eagle's medium (DMEM) supplemented with $10 \%$ fetal bovine serum (FBS) and penicillin-streptomycin $(50 \mathrm{U} / \mathrm{ml}$; Invitrogen; Thermo Fisher Scientific, Inc., Waltham, MA, USA).
Tissue collection. All molecular analyses were carried out on primary biopsies. Tissues were formalin-fixed and embedded in paraffin. Pathologists confirmed the existence of at least $80 \%$ tumor cells in the clinical specimens.

Ethics statements. The Instituto Nacional de Cancerologia (Mexico) provided the ovarian tumor and normal tissue collection. The Instituto Nacional de Cancerologia (Mexico) ethics committee approved the protocols concerning the use of human tissues. A signed informed form consent was obtained from each participant or a representative prior to release for research use.

RNA isolation from FFPE. Formalin-fixed paraffinembedded (FFPE) tissues were obtained from patients with ovarian cancer. Total RNA was isolated using the RNeasy FFPE kit (Qiagen, Valencia, CA, USA) according to the manufacture's protocol. Briefly, 5-10 FFPE sections of $10 \mu \mathrm{m}$ were incubated two times in xylene for $1 \mathrm{~h}$ at $63^{\circ} \mathrm{C}$ for deparaffinization. Then, total RNA was extracted according to the manufacturer's protocol. In addition, total RNA from SKOV-3 cells was isolated using the TRIzol protocol (Ambion, Austin, TX, USA), and concentration and purity were evaluated by spectrophotometry (NanoDrop Technologies, Wilmington, DE, USA) followed by $1 \%$ agarose-formaldehyde gel electrophoresis.

Reverse transcription and real-time polymerase chain reaction. Quantitative real-time RT-PCR (qRT-PCR) analysis for miRNA expression was performed using the TaqMan MicroRNA Assay kits (Assay ID 001178; Thermo Fisher Scientific, Inc.). Total RNA (100 ng) was reverse transcribed using a looped-RT specific primer targeting the let-7d-3p mature sequence CUAUACGACCUG CUGCCUUUCU, dNTPs (100 mM; New England Biolabs, Ipswich, MA, USA), reverse transcriptase MultiScribe (50 U/ $\mu$ l; Thermo Fisher Scientific, Inc.), 10X buffer, RNase inhibitor $(20 \mathrm{U} / \mu 1$; Promega, Madison, WI, USA) and $4.16 \mu$ l RNase-free water. Retrotranscription reaction (1:15) was mixed with master mix TaqMan (Universal PCR Master Mix, No AmpErase UNG, 2X; Thermo Fisher Scientific, Inc.), and the corresponding specific TaqMan PCR probe. PCR reaction was performed in a GeneAmp System 9700 (Applied Biosystems, Foster City, CA, USA) as follows: $95^{\circ} \mathrm{C}$ for $10 \mathrm{~min}$, and 40 cycles at $95^{\circ} \mathrm{C}$ for $15 \mathrm{sec}$ and $60^{\circ} \mathrm{C}$ for $1 \mathrm{~min}$. Tests were normalized using RNU44 as control.

let-7d-3p inhibition and scramble transfection in SKOV-3 cells. Let-7d-3p (90 nM) inhibitor (MH10785; Thermo Fisher Scientific, Inc.) and scramble (30 nM) sequence (AM17110, Thermo Fisher Scientific, Inc.) were used as negative control (https://www.thermofisher.com/order/genome-database/details/ mirna/MC10785). Both, the inhibitor and scramble sequences were individually transfected into SKOV-3 cells using siPORT amine transfection agent (Ambion). Briefly, antagomiR let-7d-3p and scramble were added to wells containing $1 \times 10^{7}$ SKOV-3 cells and incubated for $48 \mathrm{~h}$. Then, total RNA was extracted using Trizol and efficacy of antagomiR treatment in endogenous let-7d-3p downregulation was evaluated by qRT-PCR using specific stem-looped RT oligonucleotide and TaqMan probe 
(ID: 001178; Thermo Fisher Scientific, Inc.) as implemented in the TaqMan MicroRNA Assays protocol.

Cell migration and invasion assays. SKOV-3 cells $\left(1 \times 10^{5}\right)$ treated with let-7d-3p antagomiR (90 nM), or scramble sequence $(30 \mathrm{nM})$ were seeded in triplicate in a 6-well plate and grown to $80 \%$ confluence. Twenty-four hours post-transfection a vertical wound was traced in the cell monolayer. After 12 and $24 \mathrm{~h}$, cells were fixed with $4 \%$ paraformaldehyde and the scratched area was quantified. For migration assays, SKOV-3 cells $\left(1 \times 10^{5}\right)$ were transfected with let-7d-3p antagomiR or scramble sequence, and then transferred to $0.5 \mathrm{ml}$ serum-free medium and placed in the upper Transwell chambers (Corning Inc., Corning, NY, USA), whereas the lower chamber was loaded with $0.8 \mathrm{ml}$ medium containing $10 \%$ FBS. The total number of cells that migrated into the lower chamber was manually counted after $24 \mathrm{~h}$ incubation at $37^{\circ} \mathrm{C}$. Experiments were performed three times by triplicate and results are expressed as mean $\pm \mathrm{SD}$. $\mathrm{P}<0.05$ was considered as statistically significant.

Cell proliferation assays. For cell proliferation studies, the MTT reagent [(3-(4,5-dimethylthiazol-2-yl)-2,5-diphenyltetrazolium bromide] was added to SKOV-3 cells $\left(1 \times 10^{5}\right)$ and incubated for $3.5 \mathrm{~h}$ at $37^{\circ} \mathrm{C}$. Then, dissolution buffer (99\% isopropanol, $0.3 \% \mathrm{HCl}, 0.7 \% \mathrm{NP}-40$ ) was added to cells and incubated for $15 \mathrm{~min}$. Absorbance was recorded at 24 and $48 \mathrm{~h}$ using a spectrophotometer (570-630 nm). Data were analyzed using the BioStat software.

Fluorescence-activated cell sorting assays (FACS). SKOV-3 cells $\left(2 \times 10^{5}\right)$ were seeded by triplicate in a 6 -well plate and treated for $48 \mathrm{~h}$ as follows: i) siPORT transfection agent (mock); ii) scramble sequence (negative control, $30 \mathrm{nM}$ ); and iii) let-7d-3p antagomiR (90 nM). Then, carboplatin $(50 \mu \mathrm{M})$ was added to let-7d-3p inhibitor-transfected cells or alone in non-transfected cells and incubated for $24 \mathrm{~h}$. Then, cells were harvested, washed twice with PBS $1 \mathrm{X}$, resuspended in $100 \mu \mathrm{l}$ buffer (10 mM HEPES, $140 \mathrm{mM} \mathrm{NaCl}, 2.5 \mathrm{mM}$ $\mathrm{CaCl}_{2}$ ), and processed following the manufacturer's instructions (Annexin V-FLUOS staining kit; Roche Diagnostics, Basel, Switzerland). Briefly, cells were stained with $2 \mu \mathrm{l}$ Annexin V-FITC and $2 \mu \mathrm{l}$ propidium iodide (PI) mixed with $100 \mu \mathrm{l}$ incubation buffer for $15 \mathrm{~min}$, washed with $500 \mu \mathrm{l}$ binding buffer and resuspended in $300 \mu \mathrm{l}$ PBS 1X. Apoptosis events were analyzed on the FACSCalibur flow cytometer (BDIS; Becton-Dickinson, Franklin Lakes, NJ, USA). Annexin V and PI emissions were detected in the FL-1 and FL-2 channels, respectively. For each sample, data from 20,000 cells were acquired in list mode on logarithmic scales. Data were analyzed using the Summit V4.3 software and results were represented as the total percentage of apoptotic cells as the sum of both early and late phases of apoptosis (Annexin V-FITC-positive). Assays were performed by triplicate and data was expressed as mean $\pm \mathrm{SD}$. $\mathrm{P}<0.05$ was considered as statistically significant.

Prediction of gene targets and Gene Ontology (GO) analysis. miRNA target genes were predicted using TargetScan and PicTar software. Only gene targets predicted by the two algorithms were included in further analysis. Cellular pathways
Table I. Clinical and pathological features of ovarian cancer patients treated with neoadjuvant chemotherapy $(n=34)$.

Characteristics No. of patients $(\%)$

Histological subtypes

Serous papillary-high grade

$23(67.6)$

Serous papillary-low grade

Endometrioid

6 (17.6)

Mucinous

$3(9.0)$

Clear cell

$1(3.0)$

FIGO staging

I

III

4 (11.7)

$15(44.1)$

15 (44.1)

Tumor grade

1

4 (11.7)

2

$3(9.0)$

3

27 (79.4)

Metastasis status

No metastasis

$16(47.0)$

Metastasis

$18(53.0)$

Response to therapy

Partial

$12(35.2)$

Stable

$2(6.0)$

Complete

$17(50)$

Clinical progression

$1(3.0)$

Unknown

$2(6.0)$

Recurrence

Yes

$24(70.5)$

No

6 (17.6)

Unknown

4 (11.7)

Initial CA125, U/ml

$<35$

$3(9.0)$

$35-65$

$3(9.0)$

$>65$

$28(82.3)$

After treatment CA125, U/ml

$<35$

13 (38.2)

$35-65$

5 (14.7)

$>65$

$14(41.1)$

Unknown

$2(6)$

Recurrence CA125, U/ml

$<35$

$13(38.2)$

$35-65$

$6(17.6)$

$>65$

$14(41.1)$

$1(3)$

FIGO, International Federation of Gynecology and Obstetrics. CA125, cancer antigen 125 .

and processes potentially affected by let-7c-3p were predicted using DAVID 6.7 software.

Statistical analysis. Experiments were performed three times by triplicate and results are represented as mean \pm SD. 
A

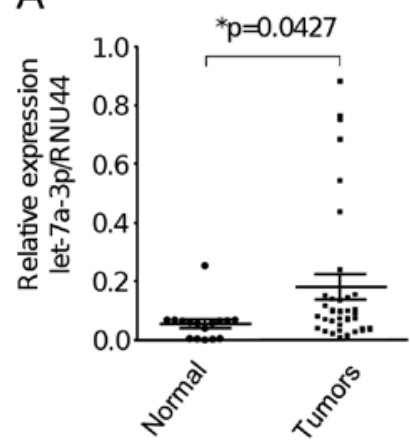

B

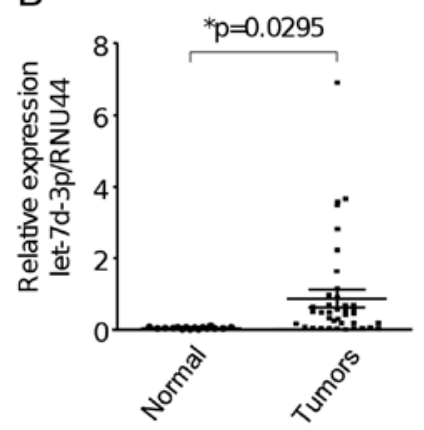

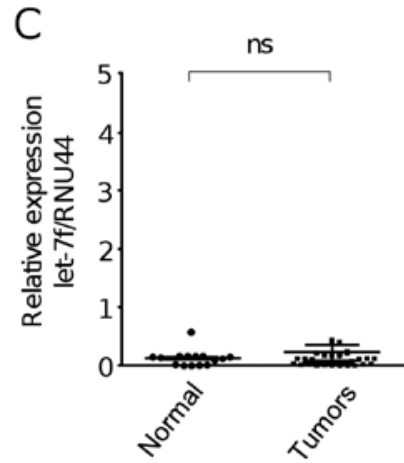

Figure 1. Expression analysis of let-7 members in ovarian tumors. Quantitative RT-PCR assays for (A) let-7a-3p, (B) let-7d-3p, and (C) let-7f expression in normal ovarian tissues $(n=18)$ and ovarian tumors $(n=40)$. Relative expression was calculated by the $2^{-\Delta \Delta C t}$ method. Data were normalized with the endogenous small-nucleolar RNU44. Data represent the mean of three independent experiments \pm SD. ns, not significant.

One-way analysis of variance (ANOVA) followed by Tukey's test were used to compare the differences between means. A $\mathrm{P}<0.05$ was considered as statistically significant.

\section{Results}

Clinical and pathological characteristics of the EOC patients. Ovarian tumor samples were collected between January 2010 and September 2012 from 34 patients diagnosed with EOC who underwent carboplatin/paclitaxel neoadjuvant treatment followed by debulking surgery at Instituto Nacional de Cancerologia. Tumor tissues were histologically analyzed by a pathologist to confirm at least $80 \%$ of tumor cells and then processed for total RNA isolation for downstream analysis. An overview of the major clinical and pathological features of tumors and patients included in the present study is provided in Table I. The average age of the patients at surgery was 52.1 years (range 35-74). Disease stage and tumor grade were classified according to the International Federation of Gynecology and Obstetrics (FIGO) and the World Health Organization (WHO) criteria, respectively. The majority of patients were diagnosed with stage III (44.1\%) and IV (44.1\%) disease, whereas $11.7 \%$ were at stage I. Tumor grade 1,2 and 3 were found in 4,3 , and 17 women, respectively. The most common histology was serous papillary high grade (67.6\%), and the remaining was endometrioid (17.6\%), mucinous (9.0\%), serous papillary low grade (3.0\%), and clear cell (3.0\%). At the time of diagnosis $16(47 \%)$ patients showed no metastasis whereas $18(53.0 \%)$ had distal metastasis. The median followup time was 34 months, ranging from 0.6 to 71 months. After neoadjuvant chemotherapy 17 patients $(50 \%)$ were classified as complete responders and $2(6 \%)$ as stable, whereas 12 (35.2\%) had a partial response. Response was monitored by measuring the levels of cancer antigen 125 (CA125 or mucin 16) and CT scan. CA125 is a well-accepted protein marker found on the surface of ovarian cancer cells and diverse types of cancer. Serial measurements of CA125 were routinely used to monitor tumor response and survival during chemotherapy.

let-7 miRNA expression is deregulated in ovarian tumors. In order to study the expression of members of the let-7 family in primary EOC tumors and normal ovarian tissues, we used stem-loop qRT-PCR as implemented in MicroRNA assay

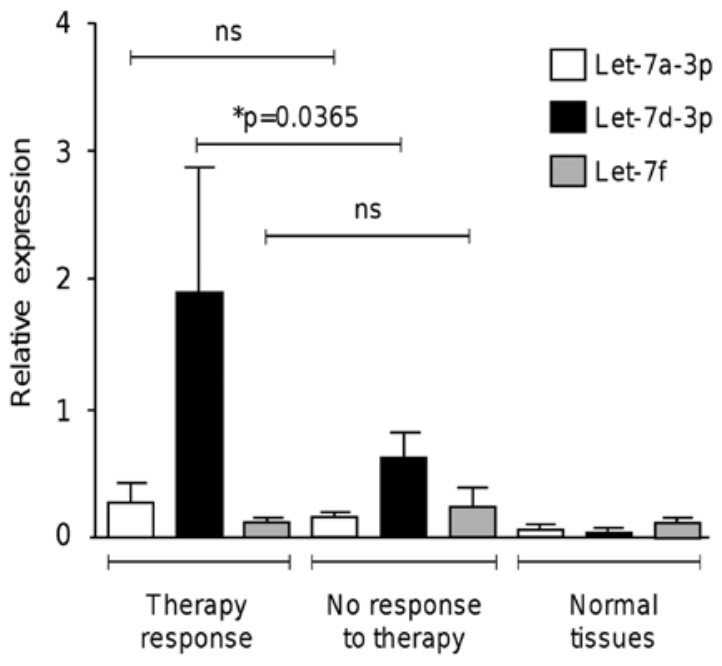

Figure 2. Expression analysis of let-7 members in patients with or without clinical response to carboplatin/paclitaxel neoadjuvant therapy. Quantitative RT-PCR analysis of expression of let-7 members was performed using MicroRNAs Taqman Assays in the group of patients achieving response $(n=12)$, and no-response $(n=22)$ to chemotherapy. Normal ovarian tissues $(n=24)$ were used as control. The relative expression of miRNAs was calculated by the $2^{-\Delta \Delta \mathrm{Ct}}$ method using RNU44 as an internal control. Bars represent the mean of three independent experiments \pm S.D. ${ }^{*} \mathrm{P}<0.05$; ns, not significant.

protocol (Thermo Fisher Scientific, Inc.). After comparative $2^{-\Delta \Delta C t}$ analyses we found that let-7a-3p and let-7d-3p were significantly $(\mathrm{P}<0.05)$ upregulated in ovarian tumors $(\mathrm{n}=40)$ in comparison to normal ovarian tissues $(n=18)$ (Fig. 1A and B). In contrast, no significant change in the expression of let-7f between the groups was found (Fig. 1C).

Upregulation of let-7d-3p is associated with a response to neoadjuvant therapy. A high percentage of patients diagnosed with locally advanced ovarian cancer have an unfavorable response to conventional treatment. This situation is aggravated as no useful molecular predictors for therapy response are currently used in clinical practice (14). We aimed to determine whether let-7 miRNA expression is associated with response to neoadjuvant therapy. We analyzed by qRT-PCR the expression of let-7 in ovarian cancer patients that had achieved a positive response and no response to carboplatin/paclitaxel 
A

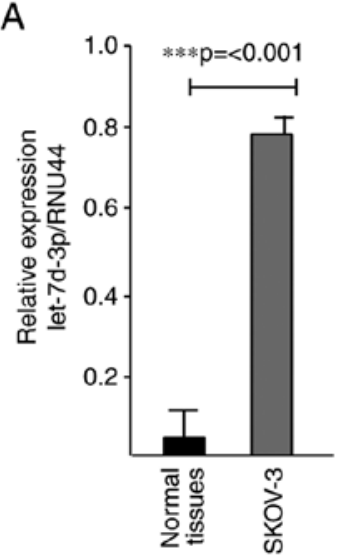

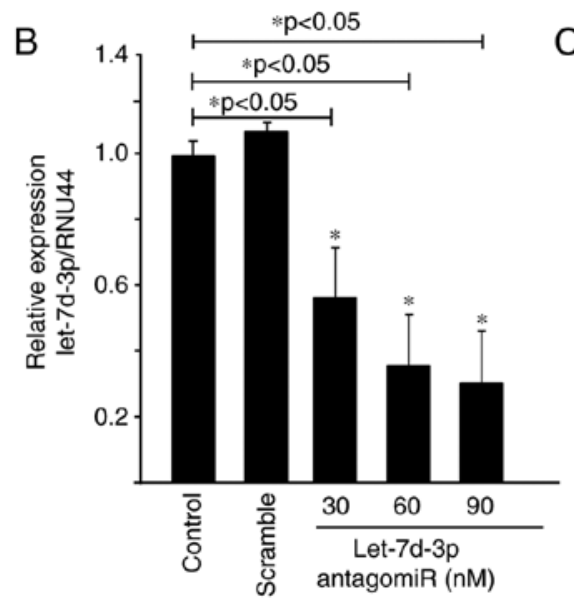

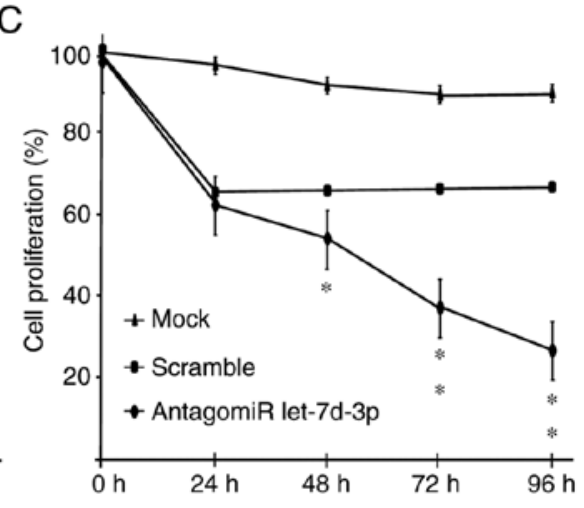

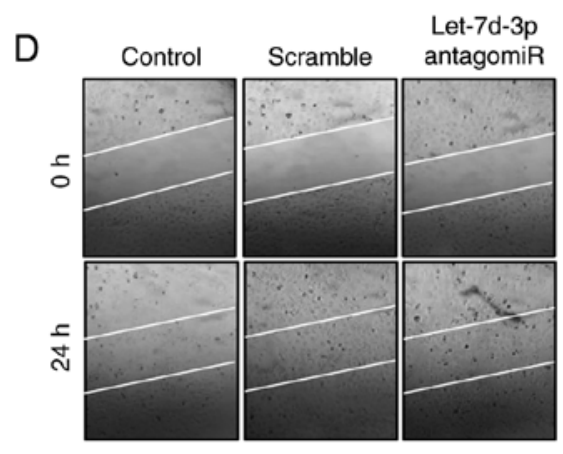

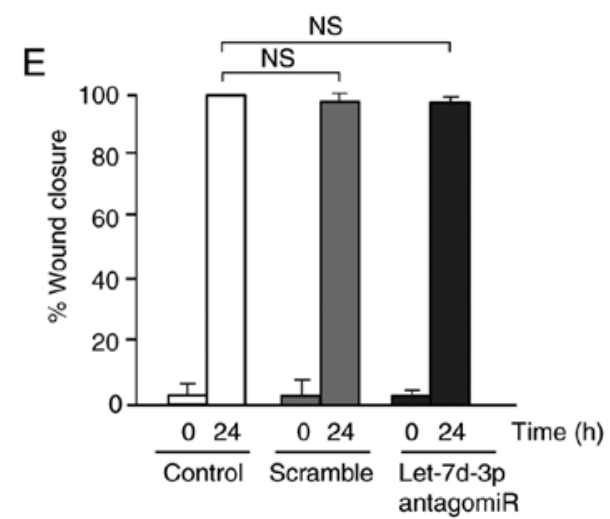

Figure 3. let-7d-3p inhibition impairs cell proliferation but not cell migration. (A) Quantitative RT-PCR assays for let-7d-3p expression in SKOV-3 ovarian cancer cells relative to normal ovarian tissues used as control. (B) Quantitative RT-PCR assays for let-7d-3p expression in SKOV-3 cells transfected with increasing concentrations (30,60 and $90 \mathrm{nM}$ ) of let-7d-3p antagomiR relative to non-transfected and scramble-transfected cells used as controls. (C) MTT cell proliferation assays of SKOV-3 cells non-transfected and transfected with scramble or let-7d-3p antagomiR $(90 \mathrm{nM})$. (D) Scratch/wound healing assays of SKOV-3 cells at $24 \mathrm{~h}$ post-transfection of scramble or let-7d-3p antagomiR. (E) Quantification of wound closure percentage of data in D. Bars represent the mean of three independent experiments $\pm \mathrm{SD}$. ${ }^{*} \mathrm{P}<0.05,{ }^{* * *} \mathrm{P}<0.001$ compared to controls. NS, not significant.

regimen. Notably, data showed that the differential expression of let-7d-3p in ovarian tumors was able to discriminate between the patients that showed response to therapy and the non-responder group $(\mathrm{P}<0.036)$ (Fig. 2). In contrast, no significant differences in the expression of let-7a-3p and let-7f between the responder and no-responder groups were found.

Effects of let-7d-3p on cell proliferation and migration. Expression analysis of let-7 members allowed us to evidence that let-7d-3p was significantly upregulated in ovarian tumors in comparison to normal ovarian tissues. To study the biological relevance of let-7d-3p we first confirmed its upregulation in SKOV-3 ovarian cancer cells relative to ovarian normal tissues (Fig. 3A). Then, knockdown of let-7d-3p expression was performed using a specific antagomiR. Data showed that transfection of increasing concentrations (30,60 and $90 \mathrm{nM})$ of let-7d-3p inhibitor significantly downregulated the endogenous let-7d-3p expression in a dose-dependent manner (Fig. 3B). We next investigated whether the forced inhibition of let-7d-3p had effects on cell proliferation in vitro. Data from the MTT assays showed that the growth rate of SKOV-3 cells transfected with let-7d-3p inhibitor $(90 \mathrm{nM})$ was significantly $(\mathrm{P}<0.05)$ decreased up to $80 \%$ in comparison with non-transfected control cells after $96 \mathrm{~h}$ (Fig. 3C). Then, we performed scratch/wound-healing assays to evaluate the contribution of let-7d-3p inhibition in cell migration. Unexpectedly, data indicated no changes in the restoration of monolayers of cells transfected with antagomiR let-7d-3p (90 nM) in comparison to non-treated and scramble transfected control cells at $24 \mathrm{~h}$ (Fig. 3D and E). Similar results in SKOV-3 cell migration were obtained using Transwell chamber assays at $24 \mathrm{~h}$ (data not shown).

Inhibition of let-7d-3p induces apoptosis. Standard treatment options for ovarian cancer patients include the use of platinum salt-based therapy. However, the effectiveness of this regimen is poor and additional therapeutic strategies are needed to improve clinical outcome. Therefore, we investigated whether the inhibition of let-7d-3p may induce apoptosis resulting in the potential sensitization of cancer cells to platinum chemotherapy. The number of apoptotic cancer cells in cultures treated with the let-7d-3p inhibitor was assessed using Annexin V-FITC assays. Our results showed that the percentage of apoptotic cells was significantly increased $(\mathrm{P}<0.05)$ from $9.8 \%$ in the non-treated control cells to $29.6 \%$ in cells treated with let-7d-3p inhibitor for $48 \mathrm{~h}$ (Fig. 4A and B). As expected, carboplatin monotherapy used as a control of cell death resulted in a marked increase in apoptotic cells $(64.5 \%)$ in comparison to mock and scramble transfected control cells. Notably, a significant increase $(\mathrm{P}<0.05)$ in cell death up to $93.8 \%$ was 
A

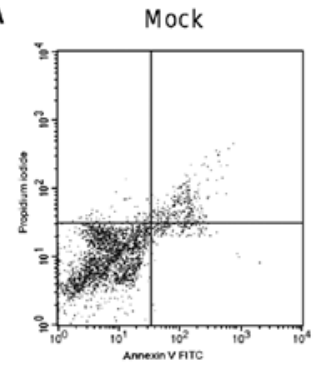

AntagomiR let-7d-3p plus carboplatin

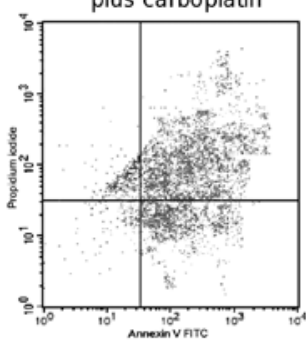

Scramble

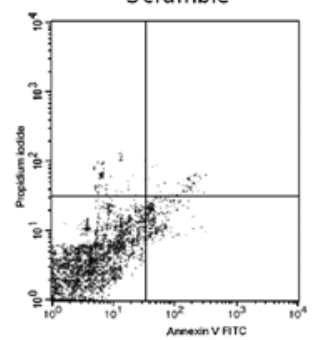

B

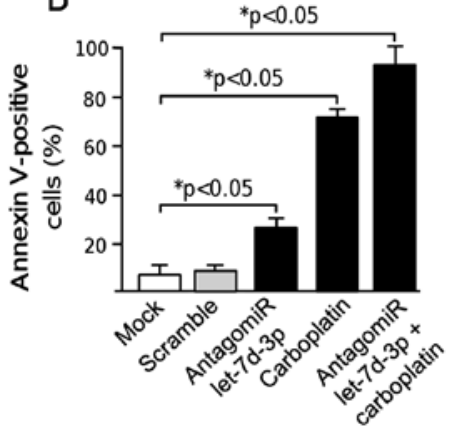

AntagomiR let-7d-3p

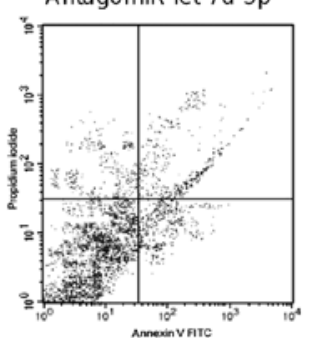

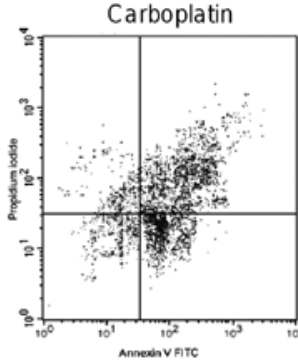

C

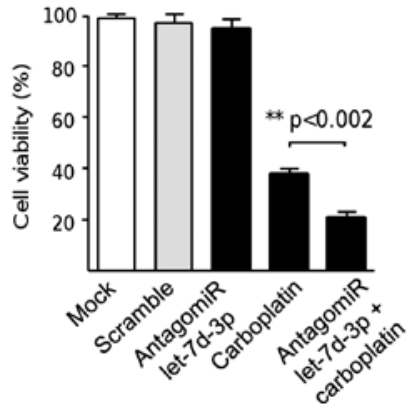

Figure 4. Inhibition of let-7d-3p induces apoptosis and enhances chemosensitivity to carboplatin. (A) Histograms obtained from FACS in Annexin V assays. SKOV-3 cells were treated with scramble $(40 \mathrm{nM})$, let-7d-3p antagomiR (90 nM), carboplatin monotherapy (50 $\mu \mathrm{M})$, and let-7d-3p antagomiR plus carboplatin for $48 \mathrm{~h}$ and Annexin V-positive cells were evaluated by FACS. (B) Quantification of data obtained in panel A. (C) MTT cell viability assays in SKOV-3 cells treated as in A. Bars represent the mean of three independent experiments $\pm \mathrm{SD}$. ${ }^{*} \mathrm{P}<0.05,{ }^{* *} \mathrm{P}<0.002$ compared to the controls.

found in let-7d-3p-deficient cells treated with carboplatin in comparison to the controls indicating a synergistic effect in apoptosis exerted by antagomiR therapy (Fig. 4A and B).

let-7d-3p inhibition sensitizes ovarian cancer cells to chemotherapy. To evaluate the potential chemosensitizing effect of let-7d-3p, we next analyzed the cell viability effects of its inhibition in combination with carboplatin cytotoxic therapy. Data showed that while treatment with the let-7d-3p inhibitor (90 nM) alone slightly affected ovarian cancer cell viability, a combination of let-7d-3p plus carboplatin $(5 \mathrm{mM})$ resulted in a marked increase in cell cytotoxicity (Fig. 4C). These data suggested that let-7d-3p sensitizes SKOV-3 cells to carboplatin therapy, at least in part, by cell death induction.

Overview of the signaling pathways modulated by let-7d-3p. Prediction of let-7d-3p targets based on GO categories identified several genes involved in key cellular processes and signaling pathways related to tumor development, progression and drug-resistance including $\mathrm{ABC}$ transporters, ErbB, RAS and HIF-1 pathways (Fig. 5A and B). For example, activation of ErbB signaling contributes to chemoradiotherapy resistance phenotypes in ovarian, breast and cervical cancer, suggesting that let-7d-3p overexpression could be associated with a complete response to therapy in ovarian cancer through similar modulation of ErbB signaling (16-18). However, additional experimental data is needed to confirm this hypothesis.

\section{Discussion}

Chemotherapy, radiotherapy and surgery are the most frequently used treatment modalities for ovarian cancer (9). In ovarian cancer, surgery followed by a combination of paclitaxel and carboplatin therapy are used as the first-line agents yielding response rates of $80 \%$ (10). Unfortunately, the majority of ovarian cancer patients relapse within the first 18 months, and recurrent disease is frequently much more resistant to conventional therapy than primary tumors (13). Therefore, alternative therapeutic approaches are needed to improve patient survival and outcome. Tumor suppressor let-7 miRNAs are frequently downregulated in human cancers, and they may be useful for the prediction of the clinical response to therapy and outcome $(7,9)$. Hence, restoration of normal expression levels of let-7 may be exploited for cancer therapeutics. Here, we analyzed the expression of three members of the let-7 miRNA family (let-7a-3p, let-7d-3p and let-7f), and explored the functions of let-7d-3p in apoptosis and therapeutic response. Our data showed that let-7d-3p overexpression was able to discriminate between the ovarian cancer patients that showed response to therapy from the non-responder group. Moreover, data indicate that let-7d-3p efficiently sensitized SKOV-3 cells to carboplatin therapy, at least in part, by cell death induction. Taken altogether, these data highlighted the potential role of let-7d-3p as a novel predictor of response to platinum-based chemotherapy.

Genetic and epigenetic alterations leading to aberrant regulation of miRNA expression is known to be involved in the development of resistance to chemotherapy of human cancers (19). Notably, the prediction of let-7d-3ptargets identified a number of genes involved in signaling pathways related to drug resistance including $\mathrm{ABC}$ transporters, ErbB, RAS and HIF-1 pathways which may be related to the chemosensitizing effect of let-7d-3p in SKOV-3 cells (Fig. 5A and B). Tumor cells acquire resistance to chemotherapeutic drugs through various mechanisms including upregulation of members of the ABC transporter family (20). The relatively rapid acquisition 
A

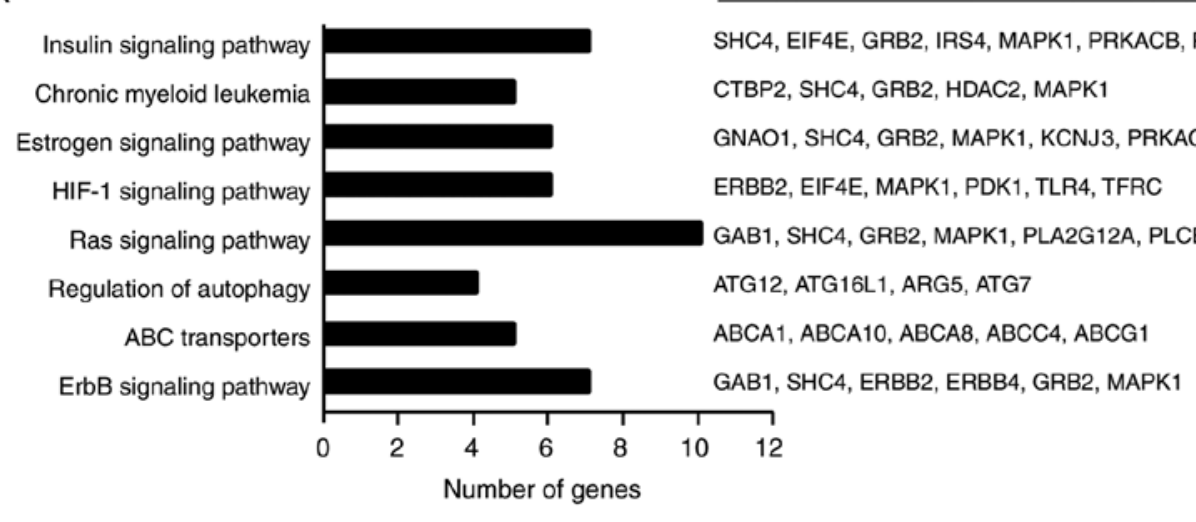

Target genes
B ERBB signaling pathway

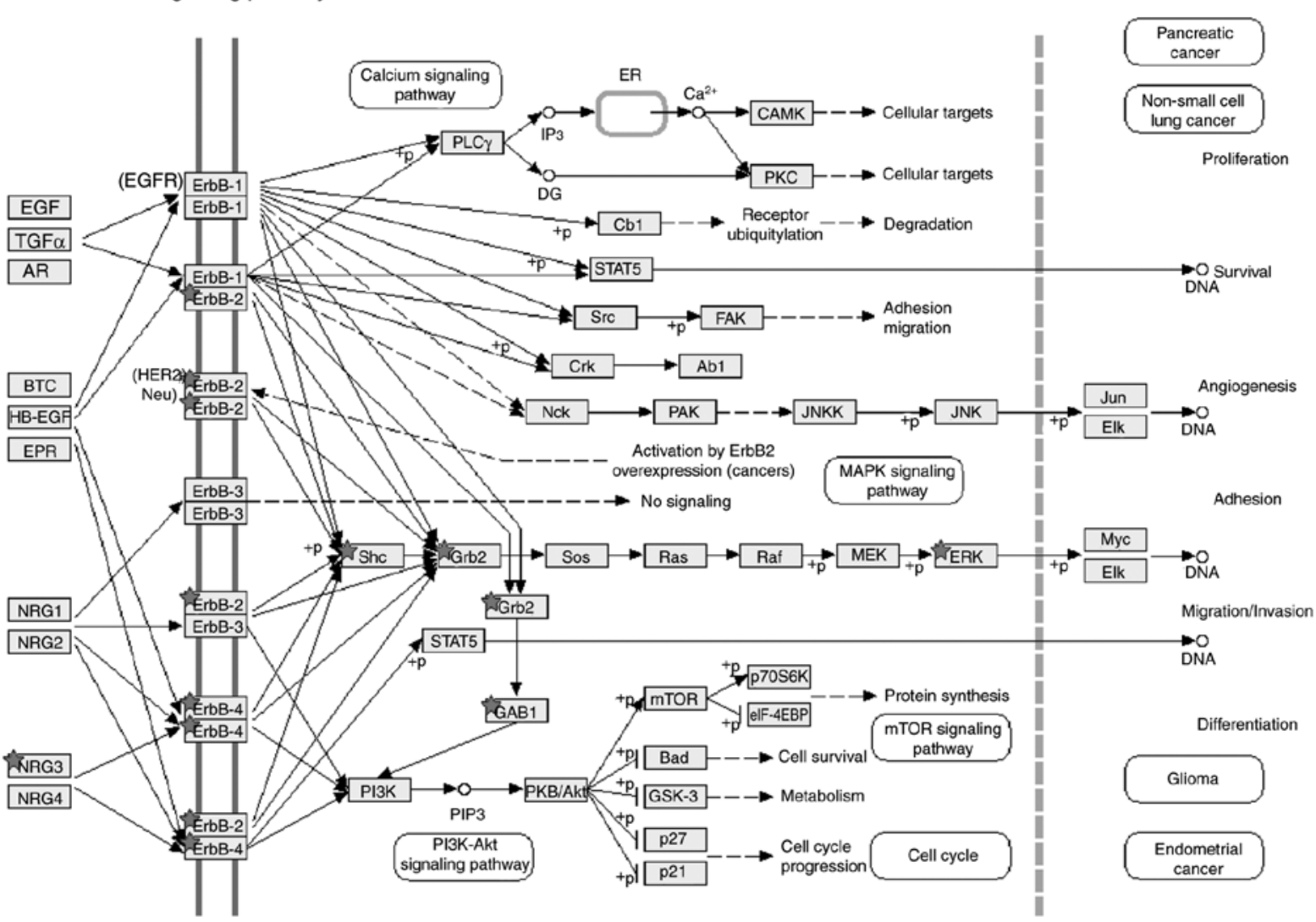

Figure 5. Genes and signaling pathways targeted by let-7d-3p. (A) Predicted let-7d-3p target genes and associated signaling pathways. (B) Predicted target genes of let-7d-3p involved in the ErbB pathway. Red stars indicate the direct predicted targets of let-7d-3p. Rectangular green boxes indicate the main genes in the ErbB signaling pathway. The pointed arrows indicate the downstream cellular processes modulated by the ErbB signaling pathways. The blunted arrows denote activation of specific proteins in ErbB signaling. The analysis was performed using KEGG pathway from David Bioinformatics tools.

of resistance to chemotherapeutic agents may be mediated by ABC transporters, MDR/MRP and P-glycoprotein; mainly they can increase efflux of drugs from cancer cells, thereby decreasing intracellular drug concentrations. Markedly, several studies have shown that miRNAs, such as miR-200c and others, can attenuate the effects of these drug-resistance components $(21,22)$. In addition, miRNAs play a critical role in the drug-resistance of tumor cells and the clinical response to cytotoxic chemotherapy in cancer (23-34). The inhibition of ABC transporters by miRNAs has been reported in several studies. For instance it was reported that both let- $7 \mathrm{i}$ and let- $7 \mathrm{~g}$ reduced $\mathrm{ABCC} 10$ expression in esophageal carcinoma (24). In addition ABCG2 was downregulated by miR-222 in squamous cell carcinoma (25). In addition, ABCA1 suppression by miR106a in lung cancer promoted cisplatin sensitivity (26). Similarly, knockdown of miR-127 enhanced the adriamycin sensitivity in glioma cells through modulation of MDR1 and MRP1 expression (27). MDR1 expression also was reduced by miR-27a through the FZD7/ $\beta$-catenin pathway resulting in increased 5-fluorouracil toxicity in hepatocellular carcinoma cells (28). On the other hand, increased miR-124 expression in renal cell carcinoma was found to promote chemosensitivity to doxorubicin by decreased of P-glycoprotein expression levels via targeting FZD5/protein kinase C (PKC) signaling (29). In addition, miR-145 upregulation enhanced the effect chemotherapeutic inhibiting P-glycoprotein through decreased 
activity of Fas signaling (30). On the other hand, miR-21 silencing in lung cancer A549/DDP cells reversed MDR by modulation of MDR-related gene expression and inhibition of AKT signaling (31).

Moreover, PI3K-AKT pathway activation could contribute to pertuzumab resistance through miR-150 downregulation in ovarian cancer (32); miR-21, miR-542-3p, miR-205 downregulation may decrease the response to trastuzumab and chemotherapy also through the PI3K pathway in breast cancer (33-35). In addition, MYC expression has been linked to tamoxifen-resistant via transcriptional regulation of the HOXB7 repressor miR-196a (36). Similarity, miR-217 low-expression levels may increase the resistance of EGFR and HER2 inhibitors through an inverse modulation of CAGE in melanoma (37).

We also observed that the Ras pathway could be impacted by let-7d-3p. The Ras pathway is also involved with acquisition of resistance to therapy and is extensively modulated by miRNAs in diverse types of cancer. For instance, miR-122 downregulation contributed to sorafenib resistance through of Ras/Raf/Erk signaling in hepatocellular carcinoma (38). In prostate cancer, miR-143 downregulation decreased the sensitivity to docetaxel by targeting the EGFR/RAS/MAPK pathway (39). Moreover, miR-3127-5p expression levels were associated with dasatinib resistance in lung cancer through the c-Abl/Ras/ERK pathway (40). Low levels of let-7b diminish the cytotoxicity of paclitaxel and gemcitabine through K-Ras mutant in several cancer types (41). Similarly, K-Ras was found to increase the 5-fluorouracil resistance through miR-224 expression in colorectal cancer (42). In ovarian cancer, miR-634 is involved with cisplatin resistance via Ras-MAPK activation (43). Finally, some reports indicate that hypoxic conditions can contribute to radiation therapy and chemotherapy resistance, and miRNAs can regulate drugresistance through direct downregulation of hypoxia inducible factor (HIF-1 $\alpha$ ) (44-47). For instance, restoration of Numb expression by inhibition of miR-182 caused HIF-1 $\alpha$ inhibition in breast cancer cells, resulting in trastuzumab resistance (45). In addition, hypoxic aggressiveness of prostate cancer cells was linked with increased expression of VEGF, IL-6 and miR-21 (46). In addition, in hepatocarcinoma, miR-338-5p sensitized cancer cells to sorafenib by targeting HIF-1 $\alpha$ (47).

In conclusion, we found that let-7d-3p, an miRNA with no previous characterized functions in ovarian cancer, was associated with apoptosis and positive response to carboplatin/paclitaxel chemotherapy in patients. Therefore, we propose that let-7d-3p could be useful as a potential molecular biomarker of the clinical response to neoadjuvant therapy in ovarian cancer patients.

\section{Acknowledgements}

We acknowledge the Autonomous University of Mexico City and CONACyT for support. RGV was recipient of a CONACYT fellowship (no. 441111).

\section{Funding}

The present study was partly funded by CONACyT (grant nos. 222335 and 233370), Mexico.

\section{Availability of data and materials}

The datasets used during the present study are available from the corresponding author upon reasonable request.

\section{Authors' contributions}

CLC, DGR and ERG conceived and designed the study. RGV, ONHDLC, YMSV, HADLV and RRP performed the experiments. ACR and SLG assisted with FACS analysis. DGR, ERG, AMG and DIO provide the tumor and normal tissues and clinical data. LAM reactived purchase and conceived the project. CLC, LAM and HAV wrote the paper.

\section{Ethics approval and consent to participate}

The Instituto Nacional de Cancerologia at Mexico provided the ovarian tumor and normal tissues collection. The corresponding ethics committee approved the protocols concerning the use of human tissues. A signed informed form consent was obtained from each participant or a representative prior to release for research use.

\section{Consent for publication}

Not applicable.

\section{Competing interests}

The authors declare that they have no competing interests.

\section{References}

1. Zhang B, Pan X, Cobb GP and Anderson TA: microRNAs as oncogenes and tumor suppressors. Dev Biol 302: 1-12, 2007.

2. Gonzalez H, Lema C, Kirken RA, Maldonado RA, VarelaRamirez A and Aguilera RJ: Arsenic-exposed keratinocytes exhibit differential microRNAs expression profile; potential implication of miR-21, miR-200a and miR-141 in melanoma pathway. Clin Cancer Drugs 2: 138-147, 2015.

3. Marin-Muller C, Li D, Bharadwaj U, Li M, Chen C, Hodges SE, Fisher WE, Mo Q, Hung MC and Yao Q: A tumorigenic factor interactome connected through tumor suppressor microRNA-198 in human pancreatic cancer. Clin Cancer Res 19: 5901-5913, 2013.

4. Ibarra I, Erlich Y, Muthuswamy SK, Sachidanandam R and Hannon GJ: A role for microRNAs in maintenance of mouse mammary epithelial progenitor cells. Genes Dev 21: 3238-3243, 2007.

5. Johnson CD, Esquela-Kerscher A, Stefani G, Byrom M, Kelnar K, Ovcharenko D, Wilson M, Wang X, Shelton J, Shingara J, et al: The let-7 microRNA represses cell proliferation pathways in human cells. Cancer Res 15: 7713-7722, 2007.

6. Kumar MS, Erkeland SJ, Pester RE, Chen CY, Ebert MS, Sharp PA and Jacks T: Suppression of non-small cell lung tumor development by the let-7 microRNA family. Proc Natl Acad Sci USA 105: 3903-3908, 2008

7. Barh D, Malhotra R, Ravi B and Sindhurani P: MicroRNA let-7: An emerging next-generation cancer therapeutic. Curr Oncol 17: 70-80, 2010 .

8. Jemal A, Siegel R, Xu J and Ward E: Cancer statistics, 2010. CA Cancer J Clin 60: 277-300, 2010.

9. Vaughan S, Coward JI, Bast RC Jr, Berchuck A, Berek JS, Brenton JD,Coukos G,Crum CC,Drapkin R,EtemadmoghadamD, et al: Rethinking ovarian cancer: Recommendations for improving outcomes. Nat Rev Cancer 11: 719-725, 2011.

10. Sandercock J, Parmar MK, Torri V and Qian W: First-line treatment for advanced ovarian cancer: Paclitaxel, platinum and the evidence. Br J Cancer 87: 815-824, 2002. 
11. Ozols RF, Bundy BN, Greer BE, Fowler JM, Clarke-Pearson D, Burger RA, Mannel RS, DeGeest K, Hartenbach EM and Baergen R; Gynecologic Oncology Group: Phase III trial of carboplatin and paclitaxel compared with cisplatin and paclitaxel in patients with optimally resected stage III ovarian cancer: A Gynecologic Oncology Group study. J Clin Oncol 21: 3194-3200, 2003.

12. Greenlee RT, Hill-Harmon MB, Murray T and Thun M: Cancer statistics, 2001. CA Cancer J Clin 51: 15-36, 2001.

13. Siddik ZH: Cisplatin: Mode of cytotoxic action and molecular basis of resistance. Oncogene 22: 7265-7279, 2003.

14. Ledermann JA, Marth C, Carey MS, Birrer M, Bowtell DD, Kaye S, McNeish I, Oza A, Scambia G, Rustin G, et al: Role of molecular agents and targeted therapy in clinical trials for women with ovarian cancer. Int J Gynecol Cancer 21: 763-770, 2011.

15. Petrillo M, Zannoni GF, Beltrame L, Martinelli E, DiFeo A, Paracchini L, Craparotta I, Mannarino L, Vizzielli G, Scambia G, et al: Identification of high-grade serous ovarian cancer miRNA species associated with survival and drug response in patients receiving neoadjuvant chemotherapy: A retrospective longitudinal analysis using matched tumor biopsies. Ann Oncol 27: 625-634, 2016

16. Li J, Zhang Y, Gao Y, Cui Y, Liu H, Li M and Tian Y: Downregulation of HNF1 homeobox B is associated with drugresistance in ovarian cancer. Oncol Rep 32: 979-988, 2014.

17. Ye XM, Zhu HY, Bai WD, Wang T, Wang L, Chen Y, Yang AG and Jia LT: Epigenetic silencing of miR-375 induces trastuzumab resistance in HER2-positive breast cancer by targeting IGF1R. BMC Cancer 14: 134, 2014.

18. Pedroza-Torres A, Fernández-Retana J, Peralta-Zaragoza O, Jacobo-Herrera N, Cantu de Leon D, Cerna-Cortés JF, Lopez-Camarillo $\mathrm{C}$ and Pérez-Plasencia C: A microRNA expression signature for clinical response in locally advanced cervical cancer. Gynecol Oncol 142: 557-565, 2016.

19. Çalışkan M, Güler H and Bozok Çetintaş V: Current updates on microRNAs as regulators of chemoresistance. Biomed Pharmacother 95: 1000-1012, 2017.

20. Sun YL, Patel A, Kumar P and Chen ZS: Role of ABC transporters in cancer chemotherapy. Chin J Cancer 31: 51-57, 2012

21. Sui H, Cai GX, Pan SF, Deng WL, Wang YW, Chen ZS, Cai SJ, Zhu HR and Li Q: miR-200c attenuates P-gp-mediated MDR and metastasis by targeting JNK2/c-Jun signaling pathway in colorectal cancer. Mol Cancer Ther 13: 3137-3151, 2014

19. Sorrentino A, Liu CG, Addario A, Peschle C, Scambia G and Ferlini C: Role of microRNAs in drug-resistant ovarian cancer cells. Gynecol Oncol 111: 478-486, 2008

22. Samuel P, Pink RC, Brooks SA and Carter DR: miRNAs and ovarian cancer: A miRiad of mechanisms to induce cisplatin drug-resistance. Expert Rev Anticancer Ther 16: 57-70, 2016.

23. Boyerinas B, Park SM, Murmann AE, Gwin K, Montag AG Zillhardt M, Hua YJ, Lengyel E and Peter ME: Let-7 modulates acquired resistance of ovarian cancer to Taxanes via IMP-1mediated stabilization of multidrug-resistance 1. Int J Cancer 130: 1787-1797, 2012.

24. Wu K, Yang Y, Zhao J and Zhao S: BAG3-mediated miRNA let-7g and let-7i inhibit proliferation and enhance apoptosis of human esophageal carcinoma cells by targeting the drug transporter ABCC10. Cancer Lett 371: 125-133, 2016.

25. Zhao L, Ren Y, Tang H, Wang W, He Q, Sun J, Zhou X and Wang A: Deregulation of the miR-222-ABCG2 regulatory module in tongue squamous cell carcinoma contributes to chemoresistance and enhanced migratory/invasive potential. Oncotarget 6: 44538-44550, 2015.

26. Ma Y, Li X, Cheng S, Wei W and Li Y: MicroRNA-106a confers cisplatin resistance in non-small cell lung cancer A549 cells by targeting adenosine triphosphatase-binding cassette A1. Mo Med Rep 11: 625-632, 2015.

27. Feng R and Dong L: Knockdown of microRNA-127 reverses adriamycin resistance via cell cycle arrest and apoptosis sensitization in adriamycin-resistant human glioma cells. Int J Clin Exp Pathol 8: 6107-6116, 2015.

28. Chen Z, Ma T, Huang C, Zhang L, Lv X, Xu T, Hu T and Li J: MiR-27a modulates the MDR1/P-glycoprotein expression by inhibiting FZD7/ $\beta$-catenin pathway in hepatocellular carcinoma cells. Cell Signal 25: 2693-2701, 2013.

29. Long QZ, Du YF, Liu XG, Li X and He DL: miR-124 represses FZD5 to attenuate P-glycoprotein-mediated chemo-resistance in renal cell carcinoma. Tumour Biol 36: 7017-7026, 2015.
30. Zheng H, Liu Z, Liu T, Cai Y, Wang Y, Lin S, Chen J, Wang J, Wang $\mathrm{Z}$ and Jiang B: Fas signaling promotes chemoresistance in gastrointestinal cancer by up-regulating P-glycoprotein. Oncotarget 15: 10763-10777, 2014

31. Dong Z, Ren L, Lin L, Li J, Huang Y and Li J: Effect of microRNA-21 on multidrug-resistance reversal in A549/DDP human lung cancer cells. Mol Med Rep 11: 682-690, 2015.

32. Wuerkenbieke D, Wang J, Li Y and Ma C: miRNA-150 downregulation promotes pertuzumab resistance in ovarian cancer cells via AKT activation. Arch Gynecol Obstet 292: 1109-1116, 2015.

33. De Mattos-Arruda L, Bottai G, Nuciforo PG, Di Tommaso L, Giovannetti E, Peg V, Losurdo A, PerezGarcia J, Masci G, Corsi F, et al: MicroRNA-21 links epithelial-to-mesenchymal transition and inflammatory signals to confer resistance to neoadjuvant trastuzumab and chemotherapy in HER2-positive breast cancer patients. Oncotarget 10: 37269-37280, 2015.

34. Ma T, Yang L and Zhang J: miRNA-542-3p downregulation promotes trastuzumab resistance in breast cancer cells via AKT activation. Oncol Rep 33: 1215-1220, 2015.

35. Iorio MV, Casalini P, Piovan C, Di Leva G, Merlo A, Triulzi T, Ménard S, Croce CM and Tagliabue E: microRNA-205 regulates HER3 in human breast cancer. Cancer Res 69: 2195-2200, 2009.

36. Jin K, Park S, Teo WW, Korangath P, Cho SS, Yoshida T, Győrffy B, Goswami CP, Nakshatri H, Cruz LA, et al: HOXB7 Is an ER $\alpha$ cofactor in the activation of HER2 and multiple ER target genes leading to endocrine resistance. Cancer Discov 5: 944-959, 2015

37. Kim Y, Kim H, Park D, Han M, Lee H, Lee YS, Choe J, Kim YM and Jeoung D: miR-217 and CAGE form feedback loop and regulates the response to anti-cancer drugs through EGFR and HER2. Oncotarget 7: 10297-10321, 2016.

38. Xu Y, Huang J, Ma L, Shan J, Shen J, Yang Z, Liu L, Luo Y, Yao C and Qian C: MicroRNA-122 confers sorafenib resistance to hepatocellular carcinoma cells by targeting IGF-1R to regulate RAS/RAF/ERK signaling pathways. Cancer Lett 371: 171-181, 2016.

39. Xu B, Niu X, Zhang X, Tao J, Wu D, Wang Z, Li P, Zhang W, Wu H, Feng N, et al: miR-143 decreases prostate cancer cells proliferation and migration and enhances their sensitivity to docetaxel through suppression of KRAS. Mol Cell Biochem 350: 207-213, 2011.

40. Sun Y, Chen C, Zhang P, Xie H, Hou L, Hui Z, Xu Y, Du Q, Zhou X, Su B and Gao W: Reduced miR-3127-5p expression promotes NSCLC proliferation/invasion and contributes to dasatinib sensitivity via the c-Abl/Ras/ERK pathway. Sci Rep 4: 6527,2014

41. Dai X, Jiang Y and Tan C: Let-7 sensitizes KRAS mutant tumor cells to chemotherapy. PLoS One 10: e0126653, 2015.

42. Amankwatia EB, Chakravarty P, Carey FA, Weidlich S, Steele RJ, Munro AJ, Wolf CR and Smith G: MicroRNA-224 is associated with colorectal cancer progression and response to 5-fluorouracil-based chemotherapy by KRAS-dependent and -independent mechanisms. Br J Cancer 112: 1480-1490, 2015.

43. van Jaarsveld MT, van Kuijk PF, Boersma AW, Helleman J, van IJcken WF, Mathijssen RH, Pothof J, Berns EM, Verweij J and Wiemer EA: $m i R$ - 634 restores drug sensitivity in resistant ovarian cancer cells by targeting the Ras-MAPK pathway. Mol Cancer 14: 196, 2015.

44. Ajduković J: HIF-1 - a big chapter in the cancer tale. Exp Oncol 38: 9-12, 2016.

45. Sajadimajd S, Yazdanparast R and Akram S: Involvement of Numb-mediated HIF-1 $\alpha$ inhibition in anti-proliferative effect of PNA-antimiR-182 in trastuzumab-sensitive and -resistant SKBR3 cells. Tumour Biol 37: 5413-5426, 2016

46. Bao B, Ahmad A, Kong D, Ali S, Azmi AS, Li Y, Banerjee S, Padhye S and Sarkar FH: Hypoxia induced aggressiveness of prostate cancer cells is linked with deregulated expression of VEGF, IL- 6 and miRNAs that are attenuated by CDF. PLoS One 7: e43726, 2012.

47. Xu H, Zhao L, Fang Q, Sun J, Zhang S, Zhan C, Liu S and Zhang Y: MiR-338-3p inhibits hepatocarcinoma cells and sensitizes these cells to sorafenib by targeting hypoxia-induced factor $1 \alpha$. PLoS One 9: e115565, 2014 